Gut and Liver, Vol. 13, No. 1, January 2019, pp. 3-4

\title{
Is EndoFLIP Useful for Predicting Clinical Outcomes after Peroral Endoscopic Myotomy in Patients with Achalasia?
}

\author{
Gwang Ha Kim \\ Department of Internal Medicine, Pusan National University School of Medicine, and Biomedical Research Institute, Pusan National University \\ Hospital, Busan, Korea
}

See "Assessment of Clinical Outcomes after Peroral Endoscopic Myotomy via Esophageal Distensibility Measurements with the Endoluminal Functional Lumen Imaging Probe" by In Kyung Yoo, et al. on page 32, Vol. 13. No. 1, 2019

The esophagogastric junction (EGJ) is a complex anatomic and functional zone that has paradoxical actions, such as allowing food passage into the stomach after swallowing and maintaining a tightly closed anti-reflux barrier. The delicate EGJ balance requires the harmonious interactions of the lower esophageal sphincter (LES), crural diaphragm, luminal geometry, and the inherent mechanical characteristics of the EGJ. ${ }^{1}$ Highresolution manometry (HRM) and timed barium esophagography (TBE) are the two main modalities used to measure the surrogate parameters of EGJ opening, such as deglutitive EGJ relaxation pressure and bolus retention. Recently, the endoluminal functional lumen imaging probe (EndoFLIP) was developed to evaluate the pathogenesis of EGJ diseases. The EndoFLIP uses impedance planimetry for real-time measurement of the EGJ cross-sectional area (CSA), the corresponding pressure, and EGJ distensibility. EGJ distensibility can be expressed as the distensibility index (DI), defined as the CSA divided by the pressure, and represents the degree of impaired LES relaxation.

Achalasia is a primary esophageal motility disorder associated with functional obstruction at the EGJ level due to defective LES relaxation. Current treatment options for achalasia focus on the relief of this functional obstruction. Of these treatments, peroral endoscopic myotomy (POEM), is an emerging minimally invasive treatment modality that provides an alternative to surgical myotomy. Recent studies have shown that POEM is a highly effective treatment for achalasia in terms of both symptomatic relief and improved esophageal physiology.

In practice, predicting post-treatment outcomes is important, including after POEM. However, there is a paucity of objective modalities for determining the extent of the myotomy and the post-POEM relaxation impairment. In the case of pneumatic dilation, a low post-dilation LES pressure $(<10 \mathrm{~mm})$ is reported to be predictive of favorable long-term outcomes. ${ }^{4}$ In addition, physiologic evaluation of the EGJ opening, such as HRM-determined LES relaxation pressure and TBE-determined bolus retention, can determine post-treatment outcomes in patients with achalasia $;^{5,6}$ however, these tests are suboptimal. Evaluation of EGJ distensibility, such as EndoFLIP DI, may be another option for determining post-POEM outcomes. In a previous study, the DI was well correlated with both the Eckardt score and the HRM-determined integrated relaxation pressure. Further, the DI was significantly less than normal in patients with poor treatment responses whereas patients with good treatment responses demonstrated significantly greater EGJ distensibility than did those with poor responses. ${ }^{1}$

In this issue of Gut and Liver, Yoo et al. ${ }^{7}$ reported that posttreatment DI by EndoFLIP is useful for predicting POEM clinical outcomes in patients with achalasia. Changes in LES pressures and integrated relaxation pressures, after POEM, and a low post-treatment DI $\left(<7 \mathrm{~mm}^{2} / \mathrm{mm} \mathrm{Hg}\right)$ during volume-controlled distension (using $30 \mathrm{~mL}$ or $40 \mathrm{~mL}$ of saline) was associated with a high rate of incomplete responses, after POEM. In a multivariate analysis, a post-treatment DI $<7 \mathrm{~mm}^{2} / \mathrm{mm} \mathrm{Hg}$ was the most important predictor of an incomplete response. Then, what are the optimal clinical DI values following POEM? Predicting the clinical response to POEM and the future occurrence of posttreatment gastroesophageal reflux seems helpful. In fact, the most common adverse event after POEM is gastroesophageal

Correspondence to: Gwang Ha Kim

Department of Internal Medicine, Pusan National University School of Medicine, and Biomedical Research Institute, Pusan National University Hospital, 179 Gudeok-ro, Seo-gu, Busan 49241, Korea

Tel: +82-51-240-7869, Fax: +82-51-244-8180, E-mail: doc0224@pusan.ac.kr pISSN 1976-2283 eISSN 2005-1212 https://doi.org/10.5009/gnl18443

(a) This is an Open Access article distributed under the terms of the Creative Commons Attribution Non-Commercial License (http://creativecommons.org/licenses/by-nc/4.0) which permits unrestricted non-commercial use, distribution, and reproduction in any medium, provided the original work is properly cited. 
reflux; the prevalence of abnormal esophageal acid exposure on $\mathrm{pH}$ monitoring after POEM has been reported to be $20 \%-57 \%{ }^{8}$ In a study involving EndoFLIP in patients undergoing Heller myotomy and POEM, a final post-treatment DI of $4.5-8.5 \mathrm{~mm}^{2}$ / $\mathrm{mm} \mathrm{Hg}$ was suggested to be the ideal final DI range for achieving optimal symptomatic outcomes, that is, Eckardt scores $\leq 1$ and GerdQ scores $\leq 7 .{ }^{9}$ On the other hand, Yoo et al. ${ }^{7}$ recommend a post-treatment DI of 7-10 $\mathrm{mm}^{2} / \mathrm{mm} \mathrm{Hg}$ for predicting minimal dysphagia and minimal gastroesophageal reflux disease, after POEM. These differences may be caused by the variability in the patient numbers, clinical response definitions, and EndoFLIP timing. Moreover, comparing EndoFLIP data between centers is difficult due to the variety of results described in previous studies. ${ }^{9}$

When is the proper time to perform EndoFLIP to predict post-POEM clinical outcomes? Recent studies have shown increased EGJ distensibility either immediately post-procedure or 3 months after treatment with pneumatic dilation, laparoscopic Heller myotomy, or POEM was predictive of postoperative symptomatic outcomes. ${ }^{3,9}$ However, there are several limitations regarding the intraoperative measurement of EGJ distensibility after POEM. Because POEM procedures are performed under general anesthesia, the drugs used can influence muscle tone even though the effects of general anesthesia on EGJ distensibility are negligible. ${ }^{10}$ The injected material used during the procedure, edema or inflammation of the EGJ tissues, and pneumoperitoneum provoked by the procedure can also influence muscle tone. Additionally, the EndoFLIP catheter can produce mucosal injury. Lastly, the late occurrence of fibrosis at the site of muscle layer defect, after POEM, can decrease the muscle distensibility, influencing the real outcomes in patients. Considering these limitations on the intraoperative measurement of EGJ distensibility, performing EndoFLIP during the follow-up period would be better. In the study of Yoo et al., EndoFLIP was performed 1 month after POEM.

EndoFLIP can provide a more direct measurement of EGJ function than either HRM or TBE, and the post-treatment DI seems to be useful for predicting treatment responses and gastroesophageal reflux, after POEM, in patients with achalasia. However, because HRM can characterize the pattern of esophageal contractility and quantify impaired EGJ relaxation and because TBE provides information on esophageal anatomy and bolus retention, both are also useful for assessing treatment efficacy. In fact, EndoFLIP, HRM, and TBE are complementary modalities; therefore, the combined use of these modalities will provide more detail and more accurate information for predicting the clinical outcomes, after POEM, in patients with achalasia.

\section{CONFLICTS OF INTEREST}

No potential conflict of interest relevant to this article was reported.

\section{REFERENCES}

1. Pandolfino JE, de Ruigh A, Nicodème F, Xiao Y, Boris L, Kahrilas PJ. Distensibility of the esophagogastric junction assessed with the functional lumen imaging probe $\left(\mathrm{FLIP}^{\mathrm{TM}}\right)$ in achalasia patients. Neurogastroenterol Motil 2013;25:496-501.

2. Ngamruengphong S, von Rahden BH, Filser J, et al. Intraoperative measurement of esophagogastric junction cross-sectional area by impedance planimetry correlates with clinical outcomes of peroral endoscopic myotomy for achalasia: a multicenter study. Surg Endosc 2016;30:2886-2894.

3. Verlaan T, Rohof WO, Bredenoord AJ, Eberl S, Rösch T, Fockens P. Effect of peroral endoscopic myotomy on esophagogastric junction physiology in patients with achalasia. Gastrointest Endosc 2013;78:39-44.

4. Alderliesten J, Conchillo JM, Leeuwenburgh I, Steyerberg EW, Kuipers EJ. Predictors for outcome of failure of balloon dilatation in patients with achalasia. Gut 2011;60:10-16.

5. Andersson M, Lundell L, Kostic S, et al. Evaluation of the response to treatment in patients with idiopathic achalasia by the timed barium esophagogram: results from a randomized clinical trial. Dis Esophagus 2009;22:264-273.

6. Nicodème F, de Ruigh A, Xiao Y, et al. A comparison of symptom severity and bolus retention with Chicago classification esophageal pressure topography metrics in patients with achalasia. Clin Gastroenterol Hepatol 2013;11:131-137.

7. Yoo IK, Choi SA, Kim WH, Hong SP, Cakir 00, Cho JY. Assessment of clinical outcomes after peroral endoscopic myotomy via esophageal distensibility measurements with the endoluminal functional lumen imaging probe. Gut Liver 2019;13:32-39.

8. Chiu PW, Wu JC, Teoh AY, et al. Peroral endoscopic myotomy for treatment of achalasia: from bench to bedside (with video). Gastrointest Endosc 2013;77:29-38.

9. Teitelbaum EN, Soper NJ, Pandolfino JE, et al. Esophagogastric junction distensibility measurements during Heller myotomy and POEM for achalasia predict postoperative symptomatic outcomes. Surg Endosc 2015;29:522-528.

10. Nathanson LK, Brunott N, Cavallucci D. Adult esophagogastric junction distensibility during general anesthesia assessed with an endoscopic functional luminal imaging probe (EndoFLIP $®)$. Surg Endosc 2012;26:1051-1055. 